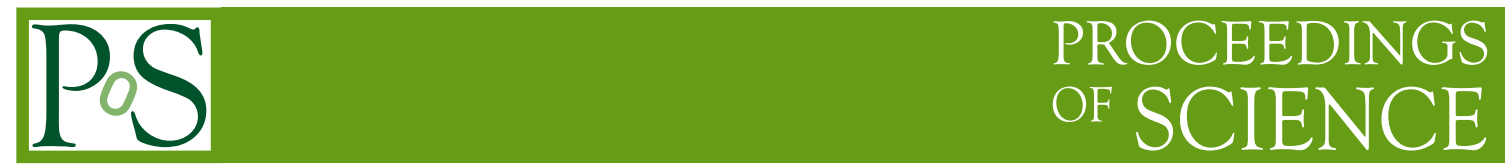

\title{
Global searches at the Tevatron
}

\section{Peter Renkel, on behalf of the D0 and CDF collaborations*}

Southern Methodist University, 75205, 6425 Boaz Lane, Dallas TX

E-mail: renkeldınaL.gov

\begin{abstract}
We present a review of global searches at the Tevatron with D0 and CDF detectors. The strategy involves splitting the data from the Tevatron into many final states and looking for signs of new physics in the high $p_{T}$ tails of various distributions using SLEUTH algorithm. CDF also utilizes Bump Hunter to search for narrow resonances in mass distributions. We analyzed 180 D0 final states, 9335 D0 distributions; 399 CDF final states and 19650 CDF distributions. No evidence of new physics is found.
\end{abstract}

European Physical Society Europhysics Conference on High Energy Physics, EPS-HEP 2009,

July 16 - 222009

Krakow, Poland

${ }^{*}$ Speaker. 


\section{Introduction}

The standard model of particle physics has been remarkably successful: all fundamental particles predicted by this model have been discovered, with the exception of the Higgs boson. Despite its success, there are strong motivations from the theory to expect new physics at energies at or just above the electroweak scale.

Generally, beyond standard model theories do not give precise energy and phase space regions to search for new physics. Motivated by this, D0[四] and CDF[ם] collaborations performed a scan over many channels to look for significant deviations from the standard model in events containing objects of high transverse momentum and invariant mass distributions.

In this searches, we widen the scope of considered final states compared to the dedicated analyses. At the same time, a sensitivity for each final state generally worse than one for the dedicated analyses.

\section{The strategy}

D0 and CDF analyzed $1 f b^{-1}$ and $3 f b^{-1}$ of data. Both D0 and CDF generate the corresponding Monte Carlo for various processes including $Z$ and $W$ boson production, diboson and $t \bar{t}$ production. D0 uses data to simulate the QCD processes, while CDF simulates these processes with Monte Carlo.

Both D0 and CDF perform a fit to obtain various Scale Factors assigned to each process. CDF does a global fit over multiple final states, while D0 considers 7 inclusive final states (dielectron, dimuon, electron + jets, muon + jets, electron + tau, muon + tau and electron + muon) to find a Scale Factor for each process in each inclusive final state.

We then define exclusive final states by considering objects such as isolated electrons, muons, taus, photons, jets, missing transverse energy and taking various combinations of those( for example electon + muon +2 jets + missing transverse energy). We impose some transverse momentum and pseudorapidity cuts on the selected objects. D0 considers only final states with at least one lepton to avoid dealing with QCD processes that are hard to simulate.

We then perform two sorts of checks in the bulk of various distributions ${ }^{1}$ on those exclusive final states. First, we perform a check on the number of events in each exclusive state; the goodness of fit is calculated by Poisson probabilities. Second, we perform a shape-only analysis of histograms within a state by calculating a Kolmogorov-Smirnov probability for the consistency of the shape with the predicted Standard Model backgrounds. Both of these numbers require additional interpretation, because of the number of trials involved. When observing many final states or many histograms, some disagreement is expected due to statistical fluctuations in the data. Thus the Poisson probability used in determining event count agreement is corrected to reflect this multiple testing. The final state probabilities converted into standard deviations before adding the trials factor correction are shown in Fig. W. D0 examines 180 final states and 9335 distributions, and CDF examines 399 final states and 19650 distributions. D0 finds 4 discrepant final states and 24 shape discrepancies - all of them seem to be discrepant due to difficulties in modeling of our detectors.

\footnotetext{
${ }^{1}$ We take out the tails of the $p_{T}$ distributions
} 
CDF doesn't observe the number uncertainties due to the nature of the global fit, and 555 shape discrepancies - all QCD related.
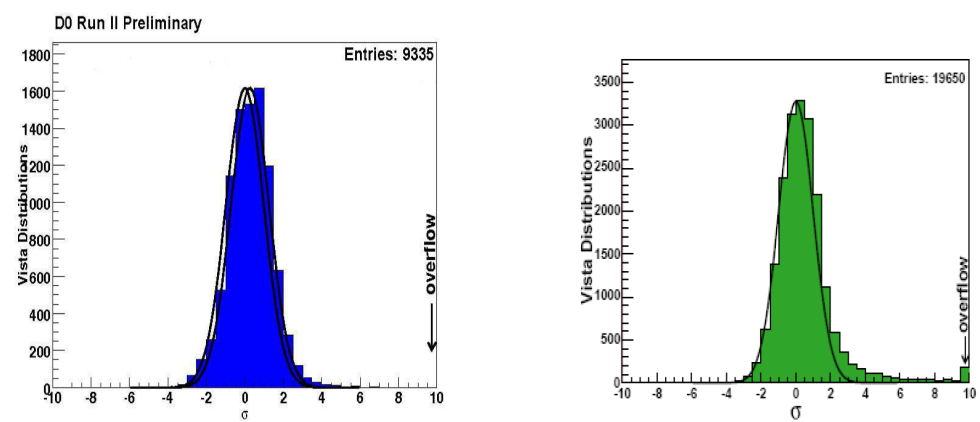

Figure 1: Vista histogram distribution $\sigma$ for $100 \%$ sample before accounting for the trials factor.

We then use SLEUTH algorithm in an attempt to systematically search for new physics as an excess in the tails of high $p_{T}$ distributions. We use a variable that adds the absolute values of the $p_{T}$ of each object in the event to the $\not_{T}$. The unclustered transverse energy is also added by CDF. We cut on the value of this variable that gives the least probability $\tilde{P}$ for the Monte Carlo to be consistent with data. We declare the state to be discrepant after trials if this probability crosses the threshold of $10^{-3}$. The Sleuth algorithm is often described as being quasi-model independent, where "quasi" refers to the assumption that the first new physics will appear as an excess of events with high- $p_{T}$ objects. Thus, Sleuth would be expected to be most sensitive to high-mass objects decaying into relatively few final state particles.

Before we proceed, we test the SLEUTH algorithm. The question we want to answer is will we be able to re-discover the top pairs had they not been discovered. For that, we remove the $t \bar{t}$ processes from our generation, and run SLEUTH. The results are presented on Fig. $\square$. The probability that the Monte Carlo after trial factors agrees with data is much smaller than the $10^{-3}$ the threshold to claim discovery.
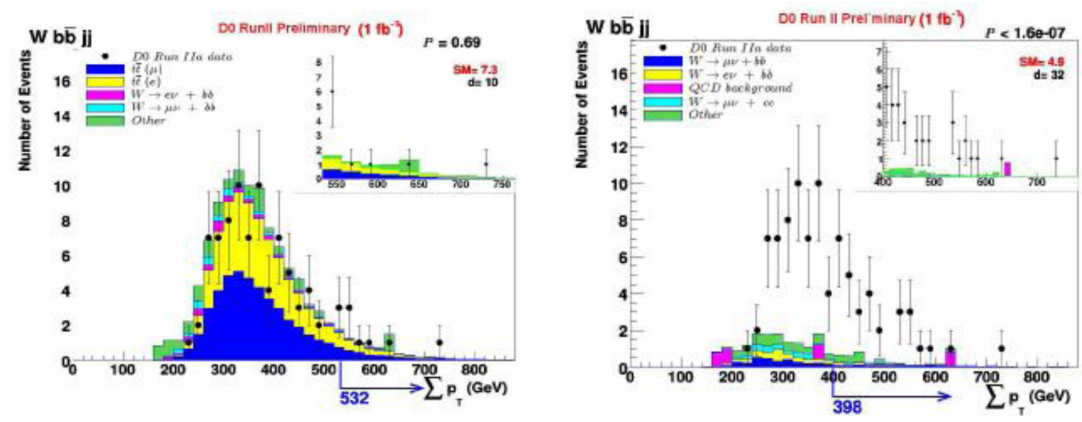

Figure 2: Sleuth plots with (left) and without (right) $t \bar{t}$ Monte Carlo for $b \bar{b}+2$ jets $+\not_{T}$.

We then run the SLEUTH on data. The most discrepant final states are given in Tab. . Only one D0 final state ' $\mu^{ \pm}+e^{\mp}+E_{T}$ ' surpasses the discovery threshold, and this seems to be related to difficulties in modeling the muon $p_{T}$ resolution. Fig. [3 shows the comparison of data and Monte Carlo for the most discrepant SLEUTH final states. 


\begin{tabular}{cc}
\hline D0 & CDF \\
\hline$l^{+} l^{6-}+E_{T}$ & $l^{+} l^{++}$ \\
\hline$l+\not_{T}$ & $l^{+} l^{6+}+j j+Z_{T}$ \\
\hline$l^{+} l^{6-}$ & $l^{+} l^{+}$ \\
\hline$l^{+} \tau^{-}+Z_{T}$ & $l^{+} l^{6-} l^{+}+B_{T}$ \\
\hline$l^{+} \tau^{-}$ & \\
\hline
\end{tabular}

Table 1: The most discrepant D0 and CDF SLEUTH final states

CDF also uses Bump Hunter to search for narrow resonances in mass distributions. We scan the mass distribution with the window of twice detector resolution for excess of data over Monte Carlo. We also require at least 5 events in such window and consistent sidebands. The most discrepant distribution after trials is shown in Fig. [1. It doesn't cross the discovery threshold.
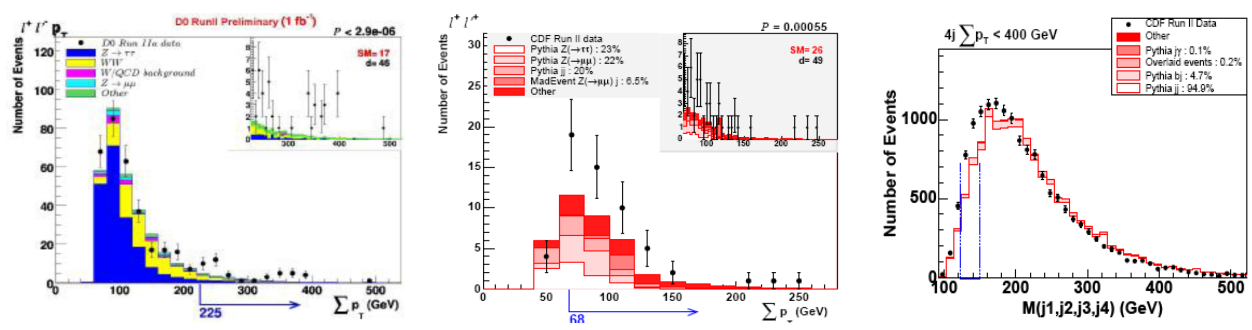

Figure 3: Sleuth plot for D0 (left) in $l^{+} l^{\natural-}$ channel and CDF (middle) in the $l+l^{\natural}+$ channel. The $\tilde{P}$ value at the top right corner of the plots is the probability before final state trials. An invariant mass of 4 jets in the 4-jet final state from CDF

\section{Conclusion}

Performing global searches with SLEUTH and Bump Hunetr, we did not find any hint of new physics in the D0 and CDF [B] data, more data has already been recorded by the experiments. As we incorporate this data set into our analysis and continue implementing improvements to our correction model, we will become much more sensitive to possible new physics.

\section{References}

[1] V.M Abazov et al., Nucl. Instrum. Methods Phys. Res. A 565, 463 (2006).

[2] A. Abulencia et al. (CDF collaboration), J. Phys. G 34, 2457 (2007).

[3] T. Aaltonen et al., Phys. Rev. D 79, 011101 (2009). 